\title{
Endovascular Stenting for Malignant Obstruction of Superior Vena Cava: A Single-Center Experience Using the Body Floss Technique
}

\author{
Dayananda Lingegowda $\quad$ Bharat Gupta ${ }^{1} \quad$ Anisha Gehani ${ }^{1} \quad$ Priya Ghosh ${ }^{1} \quad$ Sumit Mukhopadhyay ${ }^{1}$
}

${ }^{1}$ Department of Radiology, Tata Medical Center, Newtown, Kolkata, West Bengal, India

J Clin Interv Radiol ISVIR 2021;5:136-141.
Address for correspondence Dayananda Lingegowda, MBBS, DNB, Department of Radiology, Tata Medical Center, 14 Arterial Road, Newtown, Kolkata 700160, West Bengal, India (e-mail: dayanandal@gmail.com).

\begin{abstract}
Purpose Endovascular stenting is the treatment of choice for malignant obstruction of the superior vena cava (SVC) when rapid symptomatic relief is desired. Body floss technique is not routinely used during SVC stenting but has few advantages over single-access technique. We report our experience with stenting for malignant obstruction of the SVC using the body floss technique.

Materials and Methods Between March 2015 and March 2020, 25 patients (20 men, 5 women; median age, 65 years) with malignant SVC obstruction underwent endovascular stenting of the SVC. We retrospectively evaluated these patients for clinical and technical success rates, complications, recurrence-free survival, and overall survival.

Results Stent placement was successful in 24/25 patients (technical success: $96 \%$ ). Clinical success was achieved in 23/25 (92\%) patients. A mortality rate of $4 \%(1 / 25)$ was noted due to SVC rupture. Partial stent migration was noted in two patients (8\%) and was treated by placing an additional overlapping stent. Incidental early stent thrombosis was seen in two patients within 8 hours of stent placement, but these patients showed symptom relief with anticoagulation. Follow-up imaging confirmed stent patency in all patients. Late stent occlusion due to tumor progression was seen

Keywords

- superior vena cava obstruction syndrome

- SVC obstruction

- body floss technique in one patient. The primary patency rate was $88 \%(22 / 25)$. Overall median survival of 133 days was observed (range: $1-847$ days).

Conclusion Endovascular stenting of the SVC for malignant obstruction using the body floss technique is associated with high technical and clinical success, and low rate of complications.
\end{abstract}

\section{Introduction}

Superior vena cava (SVC) syndrome is a clinical condition resulting from venous hypertension secondary to SVC obstruction (SVCO). ${ }^{1}$ Malignant etiology accounts for more than $90 \%$ of cases of SVCO, with bronchogenic carcinoma

published online April 14, 2021
DOI https://doi.org/

$10.1055 / \mathrm{s}-0041-1728975$ ISSN 2457-0214 accounting for at least $50 \%^{2,3}$ SVCO is seen in up to $4 \%$ of all diagnosed bronchogenic cancers, and squamous cell carcinoma is the subtype most frequently associated with SVCO., ${ }^{1,4}$

Traditionally, malignant SVC syndrome is treated with radiotherapy and chemotherapy. ${ }^{4}$ Endovascular stenting of the SVC has gained popularity and has become the treatment

(C) 2021. Indian Society of Vascular and Interventional Radiology. This is an open access article published by Thieme under the terms of the Creative Commons Attribution-NonDerivative-NonCommercial-License, permitting copying and reproduction so long as the original work is given appropriate credit. Contents may not be used for commercial purposes, or adapted, remixed, transformed or built upon. (https://creativecommons.org/licenses/by-nc-nd/4.0/).

Thieme Medical and Scientific Publishers Pvt. Ltd. A-12, 2nd Floor, Sector 2, Noida-201301 UP, India 
of choice for acute symptomatic SVC syndrome when rapid symptomatic relief is desired. Symptoms are usually alleviated within 24 to 72 hours after SVC stenting, unlike in patients who would receive chemotherapy and/or radiotherapy where symptoms would take at least 2 to 4 weeks to subside. ${ }^{5}$ Traditionally, stenting of the SVC is achieved with a single venous access (usually femoral venous access). However, dual venous access and body floss technique have distinct advantages of superior control over the positioning and accurate deployment of the stent. We report our experience with SVC stenting for malignant SVCO using the body floss technique.

\section{Materials and Methods}

In this study, a retrospective analysis of patients who underwent stenting for malignant SVCO from 1 March 2015 to 31 March 2020 was performed. Patients were followed up from the day of the procedure until acquisition of latest information or death as an end point. All data were collected from the integrated hospital information system, or by contacting the patients telephonically wherever deemed necessary.

All patients had clinical symptoms and signs of SVC syndrome at the time of receiving requisition for stenting by the clinical team (-Table 1). All patients had either contrast-enhanced multidetector computed tomography (MDCT) or positron emission tomography CT with contrast-enhanced CT component as a part of an initial treatment workup. Length of obstruction, site of obstruction, venous diameter above and below the obstruction, Stanford grade of obstruction and presence of collaterals were noted on the cross-sectional imaging studies. ${ }^{1}$ For stable patients, histological confirmation of malignancy was obtained. For unstable patients, stenting was performed first, followed by biopsy when the clinical condition improved. All patients received preprocedural hematological evaluation for platelets, coagulation parameters, and renal function.

Informed consent was obtained prior to the procedure. All patients underwent SVC stenting under local anesthesia and monitored anesthesia care. Standard physiological monitoring of vitals including the pulse, blood pressure, oxygen saturation, and electrocardiogram was ensured during the procedure.

\section{Technique}

Venous access to right femoral vein and right internal jugular vein was obtained under ultrasound guidance and a short vascular sheath (6F) was placed. Superior vena cavogram was obtained using the jugular access to confirm the extent of venous stenosis/occlusion, collateral formation, and coexisting thrombus. Using a 5F multipurpose catheter and 0.035" standard hydrophilic guide wire, the SVCC was crossed from above using fluoroscopic guidance. After crossing the obstruction, the hydrophilic guide wire was positioned in the lower segment of the inferior vena cava and snared through the femoral sheath. Subsequently, the standard guide wire was exchanged for a $260 \mathrm{~cm}$ stiff Amplatz guide wire with the soft tip exiting the jugular sheath. Once the dual-access
Table 1 Characteristic of patients

\begin{tabular}{|c|c|}
\hline Characteristics & Values \\
\hline \multicolumn{2}{|l|}{ Sex } \\
\hline Male & 20 \\
\hline Female & 5 \\
\hline Median age & 65 y $(34-78$ y) \\
\hline \multicolumn{2}{|l|}{ Causes of superior vena cava obstruction } \\
\hline Adenocarcinoma & 11 \\
\hline Small cell carcinoma & 7 \\
\hline Squamous cell carcinoma & 2 \\
\hline Poorly differentiated carcinoma & 1 \\
\hline Sarcoma & 1 \\
\hline Metastatic disease & 2 \\
\hline No histology & 1 \\
\hline \multicolumn{2}{|l|}{ Stanford classification } \\
\hline Type 1 & 1 \\
\hline Type 2 & 11 \\
\hline Type 3 & 3 \\
\hline Type 4 & 10 \\
\hline $\begin{array}{l}\text { Primary stenting (no treatment before } \\
\text { stent) }\end{array}$ & 14 \\
\hline $\begin{array}{l}\text { Secondary stenting (received treatment } \\
\text { before stent) }\end{array}$ & 7 \\
\hline No treatment before or after stent & 4 \\
\hline Number of stents placed & 27 \\
\hline Single stent & 23 \\
\hline Double stent & 2 \\
\hline \multicolumn{2}{|l|}{ Stent diameter } \\
\hline $16 \mathrm{~mm}$ & 2 \\
\hline $18 \mathrm{~mm}$ & 8 \\
\hline $20 \mathrm{~mm}$ & 9 \\
\hline $22 \mathrm{~mm}$ & 7 \\
\hline $24 \mathrm{~mm}$ & 1 \\
\hline Average pretreatment Kishi score & 6 \\
\hline
\end{tabular}

("through-and-through" or "body-floss") was established, the femoral vein sheath was exchanged for a $11 \mathrm{~F}$ sheath and the rest of the procedure was performed through the femoral access (-Fig. 1).

The stent size was chosen at 15 to $20 \%$ more than the reference vessel diameter on MDCT. We placed $~ 60 \%$ of the length of the stent above the lesion so as to reduce the risk of central stent migration. We placed the stent in between the superior normal vein (landing zone of at least $10 \mathrm{~mm}$ margin) and SVC-right atrium junction inferiorly. When bilateral brachiocephalic veins were occluded, the stent was placed through one of the two brachiocephalic veins. Self-expanding stainless-steel stents (Wallstent, Boston Scientific, Natick, Massachusetts, United States) were used in all patients. All patients received an intravenous bolus of $70 \mathrm{IU} / \mathrm{kg}$ of heparin prior to the procedure. Pre- or poststent dilation was not routinely performed. Prestent balloon dilation was 


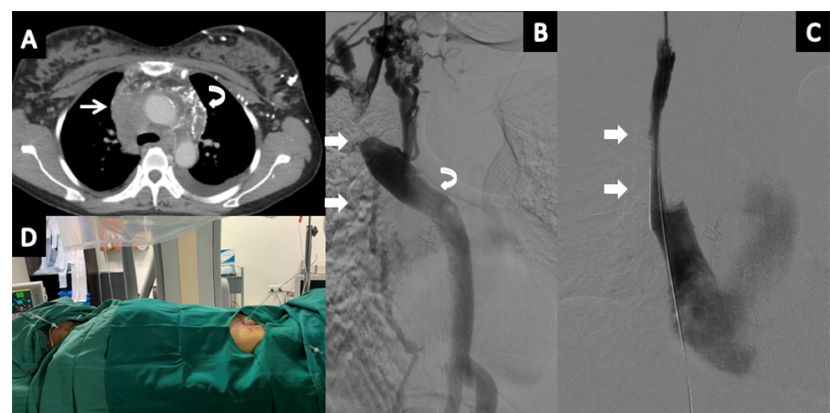

Fig. 1 Case of adenocarcinoma carcinoma with superior vena cava obstruction syndrome. (A) Computed tomography showing paratracheal mass infiltrating superior vena cava (arrow). Extensive mediastinal collateral vessels can be observed (curved arrow). (B) Venogram showing occlusion (arrows) of the superior vena cava (type 4 Stanford) with collaterals reforming the azygos vein (curved arrow). (C) Final superior vena cavogram obtained after stent placement shows free flow of contrast material through the superior vena cava (arrow) and disappearance of all venous collaterals. (D) Clinical image showing through and through access.

performed only if there was any resistance to the passage of the stent across the obstruction (5/25). Poststent dilation was performed in those cases in which there was no free flow of contrast material across the stent or in case of persistence of collateral flow even after stenting (4/25).

\section{Postprocedural Care and Follow-Up}

In the postprocedure period, subcutaneous injection of enoxaparin sodium $60 \mathrm{mg}$ was given twice daily until discharge to prevent acute stent thrombosis (average 2 days). All patients received aspirin $75 \mathrm{mg} /$ day and clopidogrel $75 \mathrm{mg} /$ day for a minimum period of 6 months following the procedure and aspirin lifelong.

After stenting, patients were evaluated for feasibility of specific antitumor treatment by their treating physicians. Patients were followed for the resolution of the clinical symptoms of SVCO. All patients underwent imaging follow-up as per the clinician's discretion to assess treatment response.

\section{Study Design}

The primary end point of our study was to assess for complete clinical success (defined by a Kishi score of $<2$ at 48 hours after stenting), partial clinical success (Kishi score between 2 and 4 at 48 hours after the procedure), or clinical failure (Kishi score above 4). Secondary end point was to evaluate symptom recurrence-free survival, time to recurrence, and overall survival. The complications of endovascular stenting were also studied. Overall survival of patients undergoing primary stenting (no prior antitumor therapy at the time of stenting but received adjuvant chemotherapy or radiotherapy after stenting), patients undergoing secondary stenting (received prior antitumor treatment), and in patients who received no antitumor treatment before or after stenting were analyzed. Partial stent migration was defined as migration from initial position, but still covering the stricture.

\section{Results}

Stent placement was successful in 24/25 patients (96\%). In one patient, due to extensive chronic thrombus and tandem lesions, we failed to cover entire length of obstruction resulting in a technical failure. Complete clinical success was seen in 23/25 (92\%) patients. Clinical failure was noted in only one patient that was due to technical failure (- Table $\mathbf{1}$ ). Mortality rate of $4 \%(1 / 25)$ was noted due to SVC rupture in one patient. Partial stent migration was noted in two patients, which was treated by placing another overlapping stent. No clinical implications were observed due to stent migration.

Incidental stent occlusion due to stent thrombosis was seen in two patients within 8 hours after stenting. Both patients clinically responded to systemic anticoagulation and showed clinical success at 48 hours. Follow-up imaging confirmed patency of the stent. One case of delayed stent occlusion (at 60 days) due to tumor progression was encountered. No secondary intervention was done in this case due to poor performance status. The primary stent patency rate was $88 \%(22 / 25)$.

Median overall survival of 133 days was observed (range: 1-847 days). Median overall survival of 149 days was observed in primary stenting patients, whereas in patients who underwent secondary stenting, the median survival was 47 days. Median survival of patients who were not fit for any cancer specific treatment after stenting was 8.5 days. Five patients were still alive at the end of the study period ( - Fig. 2 ).

\section{Discussion}

Management of thoracic malignancies with SVCO depends on multiple factors such as type of malignancy, stage of malignancy, severity of symptoms, and patient's performance status and comorbidities. ${ }^{5}$ Kishi scoring system and Yu et al's classification system serve as a guide to identify those patients who require palliative stenting at any stage of treatment. ${ }^{5,6}$ Kishi score above 4 is an indication for SVC stenting. ${ }^{7}$ If SVCO symptoms are life threatening, SVC stenting can be done before establishing the histopathological diagnosis. Stenting may not be used as the first-line treatment option for symptomatic patients with SVC syndrome caused by small cell lung cancer, non-Hodgkin lymphoma, and germ cell tumors since these tumors are chemosensitive and deserve a trial of chemotherapy. ${ }^{5,8}$

\section{Technical Success}

Our technical success rate of $96 \%$ is consistent with the technical success observed in previous studies. ${ }^{9-12}$ We failed to connect normal to normal zone in one case, due to extensive thrombus extending to both brachiocephalic veins and internal jugular vein, which eventually also showed thrombosis. In the patients with acute thrombus, catheter-directed intravascular thrombolysis can be attempted. Thrombolysis helps to reduce the thrombus load and the length of the obstruction. The thrombolysis is most effective if it is started within 2 to 5 days of onset of symptoms and tends to be ineffective 
A Overall Survival
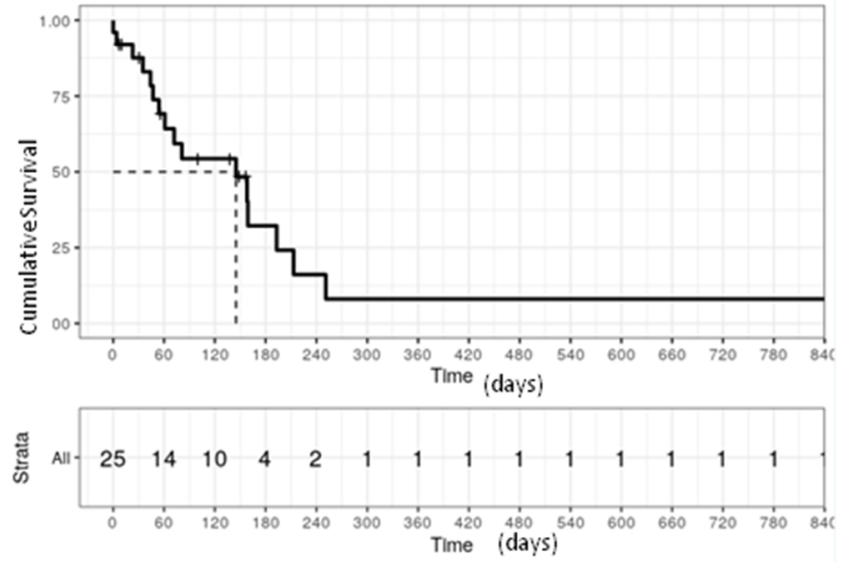

B

Overall Survival

Strata - GROUPS $=1+$ GROUPS $=2+$ GROUPS $=3$

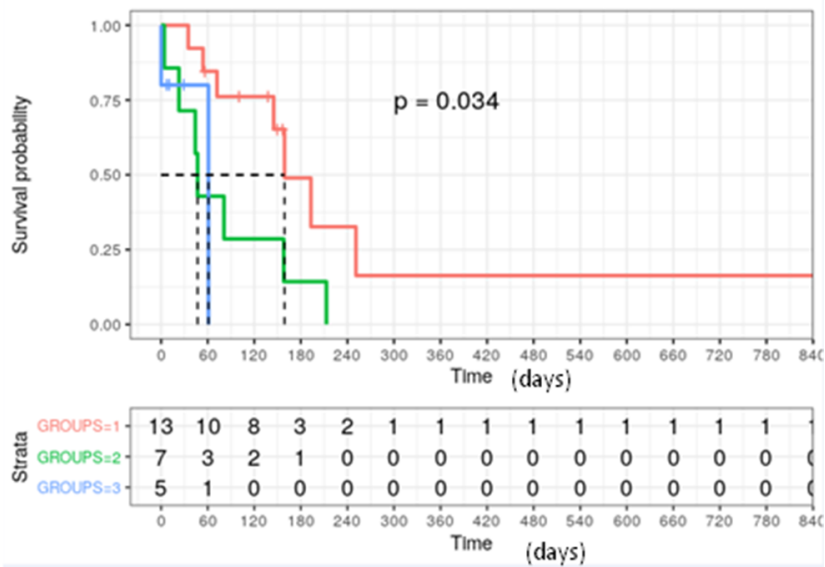

Fig. 2 Kaplan-Meier curves. (A) Overall patient survival. (B) Survival curve of group 1 (primary stenting), group 2 (secondary stenting), and group 3 (no cancer treatment after stenting).

if started after 10 days. ${ }^{4,13}$ We did not attempt thrombolysis in this case due to chronic and extensive nature of thrombus.

\section{Clinical Success}

Immediate clinical success was achieved in 92\% (23/25) of cases. If the technical failure and postprocedure mortality cases are excluded, symptomatic relief was achieved in all other cases, which translates to $100 \%$ conversion of technical success into clinical success. In comparison, available literature reports a clinical success rate of 50 to $70 \%$ with radiotherapy and takes 2 to 4 weeks for symptomatic relief., 54,15 A meta-analysis of prospective and retrospective studies from 1983 to 1997 for small cell lung cancer found that chemotherapy could achieve symptomatic relief in up to $76.9 \%$ cases. $^{5}$

\section{Unilateral versus Bilateral Stenting}

Unilateral stents were placed in all cases, even in those with bilateral brachiocephalic vein obstruction. Our study also reinforces the arguments from Dinkel et al that unilateral placement of stent will suffice irrespective of type of SVCO. ${ }^{8,16}$ Unilateral stenting is technically more simple, is more cost-effective, is associated with far lower complication, and has similar outcomes as compared with bilateral stenting. ${ }^{16}$

\section{Recurrence Rate and Reintervention}

Out of the 23 patients who underwent successful stenting without any major complication, 22 (95\%) patients were free from symptoms due to SVCO till death or up to March 2020. This is very well aligned with the primary patency rate of 86 to $93 \%$ reported in the literature. ${ }^{8}$ Two cases of immediate stent thrombosis successfully responded to routine anticoagulation therapy. One case of recurrent SVCO due to tumor progression was observed in our series. We did not perform any reintervention in this case due to the patient's poor general condition.

\section{Overall Survival}

Median survival of 133 days was noted in our series. SVC stenting patients have a dismal prognosis with median survival ranging from 1.5 to 10 months, irrespective of any treatment received. ${ }^{5}$

\section{Effect of Adjunct Chemotherapy and/or Radiotherapy on Overall Survival}

Patients who underwent primary stenting followed by chemotherapy and/or radiotherapy lived longer than patients who did not receive any treatment after stenting. Interestingly, literature suggests that patients who received chemotherapy and/or radiotherapy prior to stenting were likely to die early as compared with patients who received primary stenting. ${ }^{8}$ Similar observation was also made in our series. The most plausible explanation in favor of this would be that secondary stenting cases represent primary treatment failure with chemotherapy and/or radiotherapy (progressive disease).

\section{Mortality and Morbidity}

A single mortality due to pericardial tamponade was observed in our series (4\%), which manifested 2 hours after the procedure (-Fig. 3). The risk factors for cardiac tamponade are vascular fragility due to prior radiotherapy or chemotherapy, perforation caused by wire during crossing, excessive balloon dilation, and large stent diameter. ${ }^{17}$

Approximately, distal $3.5 \mathrm{~cm}$ of the SVC is not covered by serous pericardium. This "danger zone" should be avoided as a "landing zone" for stents, since this segment is prone for rupture. ${ }^{17}$ The stent margin is free and sharp that might protrude outside the wall particularly when stents are oriented obliquely. In our case, larger stent diameter ( $24 \mathrm{~mm}$ ) and distal end of stent in the danger zone were the likely causes of SVC rupture. 


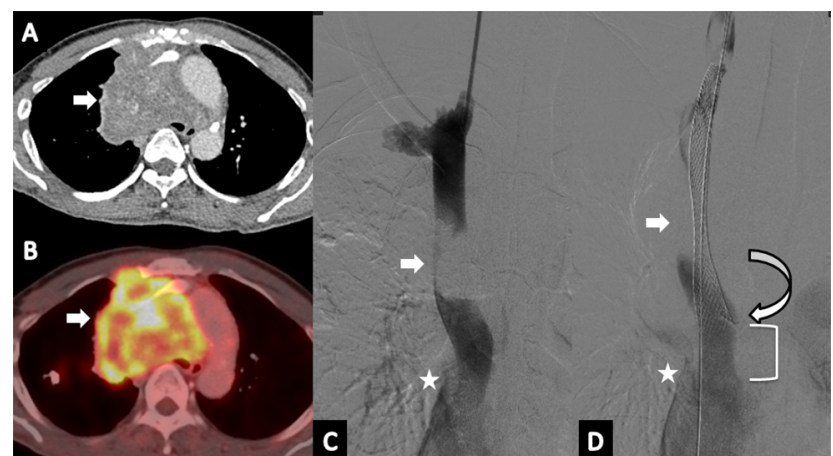

Fig. 3 Pericardial tamponade post-stenting in a case of superior vena cava (SVC) obstruction due to adenocarcinoma lung. (A, B) Computed tomography and positron emission tomography images showing large mass in right mediastinum infiltrating the SVC (arrows). (C) Venogram showing large filling defects in SVC (arrow). (D) Final superior vena cavogram obtained after stent placement $(24 \mathrm{~mm})$ showing distal end of stent (curved arrow) is above cavoatrial junction (star). Note the danger zone of SVC (bracket).

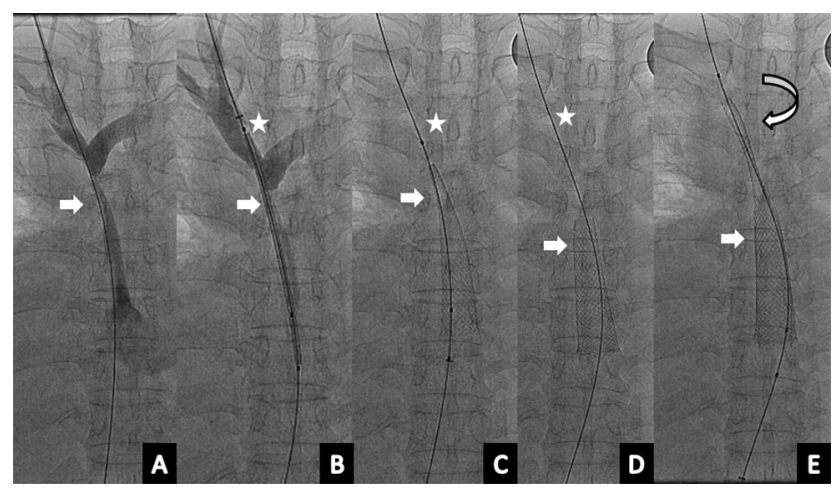

Fig. 4 Stent migration in case of adenocarcinoma with superior vena cava (SVC) obstruction syndrome. (A) Superior vena cavogram shows high-grade SVC stenosis (arrow). (B) Image shows prerelease stent position in relation to venous stenosis (arrow). The upper end of the stent is in the right brachiocephalic vein (star). (C, D) Progressive stent migration (arrow) from initial position (star). (E) Migrating stent (arrow) is stabilized using a longer stent (curved arrow).

Two cases of partial stent migration were seen in our series (8\%). This happened when we tried to place a short length Wallstent ( -Fig. 4). After differential foreshortening at the upper end, the stent migrated below the stenosis. We placed a second larger stent to anchor the migrating stent and also to cover the stenosis. Various strategies are described to manage stent migration. Retrieving the stent using a snare for a fully migrated stent is desirable. ${ }^{18}$ By avoiding routine pre- and post-balloon dilation and hoping that residual stenosis anchors the stent, stent migration can be prevented. ${ }^{18}$

Apart from giving stability and pushability during crossing the lesion, the body floss technique comes in handy in case of stent migration, since the stent is still held over the wire. ${ }^{18}$ In case of stent migration, this technique not only prevents major cardiac events by preventing stent migration to the right ventricle but it also gives sufficient time as well as stability and strong scaffold for stent retrieval. In case of SVC rupture, the body floss technique provides stable access to place the balloon tamponade/covered stent graft. Literature shows body floss technique is used only during difficult access cases. ${ }^{13}$ In our experience, routine use of body floss technique adds to the confidence of the operator during the procedure and the operator is better prepared handling the potentially fatal complications. Complication rate up to $19 \%$ is reported in the literature; hence, body floss technique can make a difference in one-fifth of patients. ${ }^{13}$

\section{Conclusion}

A high technical and clinical success is achieved with endovascular stenting of the SVC for highly symptomatic malignant SVCO. Unilateral stenting is sufficient in most cases. Body floss technique is very helpful during the procedure and helps manage stent related complications.

\section{Conflict of Interest \\ None declared.}

\section{References}

1 Lepper PM, Ott SR, Hoppe H, et al. Superior vena cava syndrome in thoracic malignancies. Respir Care 2011;56(5):653-666

2 Chen JC, Bongard F, Klein SR. A contemporary perspective on superior vena cava syndrome. Am J Surg 1990;160(2):207-211

3 Parish JM, Marschke RF Jr, Dines DE, Lee RE. Etiologic considerations in superior vena cava syndrome. Mayo Clin Proc 1981;56(7):407-413

4 Uberoi R. Quality assurance guidelines for superior vena cava stenting in malignant disease. Cardiovasc Intervent Radiol 2006;29(3):319-322

5 Straka C, Ying J, Kong F-M, Willey CD, Kaminski J, Kim DWN. Review of evolving etiologies, implications and treatment strategies for the superior vena cava syndrome. Springerplus 2016;5:229

$6 \mathrm{Yu} \mathrm{JB}$, Wilson LD, Detterbeck FC. Superior vena cava syndrome-a proposed classification system and algorithm for management. J Thorac Oncol 2008;3(8):811-814

7 Kishi K, Sonomura T, Mitsuzane K, et al. Self-expandable metallic stent therapy for superior vena cava syndrome: clinical observations. Radiology 1993;189(2):531-535

8 Lanciego C, Pangua C, Chacón JI, et al. Endovascular stenting as the first step in the overall management of malignant superior vena cava syndrome. Am J Roentgenol 2009;193(2):549-558

9 Uberoi R. CIRSE Quality Assurance Guidelines for Superior Vena Cava Stenting in Malignant Disease. CardioVasc Interv Radiol 2006;29:319-322

10 Ganeshan A, Hon LQ Warakaulle DR, Morgan R, Uberoi R. Superior vena caval stenting for SVC obstruction: current status. Eur J Radiol 2009;71(2):343-349

11 Fagedet D, Thony F, Timsit J-F, et al. Endovascular treatment of malignant superior vena cava syndrome: results and predictive factors of clinical efficacy. Cardiovasc Intervent Radiol 2013;36(1):140-149

12 Tanigawa N, Sawada S, Mishima K, et al. Clinical outcome of stenting in superior vena cava syndrome associated with malignant tumors. Comparison with conventional treatment. Acta Radiol 1998;39(6):669-674 
13 Rachapalli V, Boucher L-M. Superior vena cava syndrome: role of the interventionalist. Can Assoc Radiol J 2014;65(2):168-176

14 Ahmann FR. A reassessment of the clinical implications of the superior vena caval syndrome. J Clin Oncol 1984;2(8):961-969

15 Wilson LD, Detterbeck FC, Yahalom J. Clinical practice. Superior vena cava syndrome with malignant causes. N Engl J Med 2007;356(18):1862-1869

16 Dinkel H-P, Mettke B, Schmid F, Baumgartner I, Triller J, Do D-D. Endovascular treatment of malignant superior vena cava syndrome: is bilateral Wallstent placement superior to unilateral placement? J Endovasc Ther 2003;10(4):788-797

17 Chang S-N, Chi N-H, Lee W-J, Hsu K-L, Chiang F-T, Tseng C-D. Cardiac tamponade after stenting for superior vena cava obstruction. Zhonghua Minguo Xinzangxue Hui Zazhi 2009;25:161-164

18 Taylor JD, Lehmann ED, Belli A-M, et al. Strategies for the management of SVC stent migration into the right atrium. Cardiovasc Intervent Radiol 2007;30(5):1003-1009 